\title{
Laser de baixa potência no tratamento de manifestação exuberante da sífilis congênita
}

\author{
Low-power laser in the treatment of exuberant manifestations of congenital syphilis \\ Láser de baja potencia en el tratamiento de manifestaciones exuberantes de sífilis congénita
}

Recebido: 11/10/2021 | Revisado: 18/10/2021 | Aceito: 20/10/2021 | Publicado: 23/10/2021

Gabriel Umbelino Santana

ORCID: https://orcid.org/0000-0003-4651-5491 Hospital Universitário Maria Aparecida Pedrossian, Brasil

E-mail: gaielsantanna@gmail.com

Gabriela Pestana da Costa

ORCID https://orcid.org/0000-0001-8996-7063 Hospital Universitário Maria Aparecida Pedrossian, Brasil

E-mail: gabrielapestanacosta@gmail.com

Yara Diniz Souza

ORCID: https://orcid.org/0000-0001-7845-0130 Hospital Universitário Maria Aparecida Pedrossian, Brasil

E-mail: dinizsouzay@gmail.com

Pamela Ferreira de Sá Queiroz

ORCID: https://orcid.org/0000-0003-0912-3675

Hospital Universitário Maria Aparecida Pedrossian, Brasil

E-mail: pamelaqueiroz88@gmail.com

Regina Maria Raffaele

ORCID: https://orcid.org/0000-0002-1636-3688

Associação Beneficente Santa Casa de Campo Grande, Brasil

E-mail: reginaraffaele@hotmail.com

Leonardo Perez Faverani

ORCID: https://orcid.org/0000-0003-2249-3048

Universidade Estadual Paulista Júlio de Mesquita Filho, Brasil

E-mail: leobucomaxilo@gmail.com

Ellen Cristina Gaetti-Jardim

ORCID: https://orcid.org/0000-0003-2471-465X

Hospital Universitário Maria Aparecida Pedrossian, Brasil

E-mail: ellen.jardim@ufms.br

\begin{abstract}
Resumo
A sífilis congênita ainda é uma realidade nos países do terceiro mundo, resultando em complicações locais e sistêmicas que comprometem a qualidade de vida do paciente portador. Deste modo, é objetivo deste artigo, apresentar um caso clínico de paciente lactante, sexo masculino, de 34 dias de vida, diagnosticado com sífilis congênita. Ao exame clínico o paciente apresentou inúmeras ulcerações e lesões teciduais em boca, face e membros. Como terapia adjuvante, foi associado as condutas-padrão a aplicação do laser de baixa potência. Após 8 sessões da terapia fotodinâmica houve remissão completa do quadro clínico apresentado, devolvendo qualidade a tenra vida do paciente. Com o caso apresentado, as manifestações e complicações da sífilis ainda se mostram desafiadoras. É fundamental que a equipe multiprofissional seja capaz de diagnosticar e tratar o mais precocemente possível a sífilis congênita; o cirurgião-dentista deve ter o conhecimento das diversas manifestações clínicas dela para ajudar no diagnóstico correto e tratamento antes do começo da sintomatologia. A terapia de fotobiomodulação com laser de baixa potência pareceu acelerar satisfatoriamente o processo de reparação tecidual. A resposta do paciente ao tratamento instituído foi gratificante.
\end{abstract}

Palavras-chave: Sífilis; Sífilis congênita; Terapêutica; Terapia com Luz de Baixa Intensidade.

\begin{abstract}
Congenital syphilis is still a reality in third world countries, resulting in local and systemic complications that compromise the patient's quality of life. Thus, the aim of this article is to present a clinical case of a 34-day-old male lactating patient diagnosed with congenital syphilis. On clinical examination, the patient presented numerous ulcerations and tissue lesions in the mouth, face and limbs. As adjuvant therapy, the standard approaches were associated with the application of low-power laser. After 8 sessions of photodynamic therapy, there was complete remission of the clinical condition presented, returning quality to the patient's tender life. With the case presented, the manifestations and complications of syphilis are still challenging. It is essential that the multidisciplinary team is able to diagnose and treat congenital syphilis as early as possible; the dentist must have knowledge of the various clinical
\end{abstract}


manifestations of it to help in the correct diagnosis and treatment before the onset of symptoms. Low power laser photobiomodulation therapy appeared to satisfactorily accelerate the tissue repair process. The patient's response to the instituted treatment was gratifying.

Keywords: Syphilis; Syphilis, congenital; Therapeutics; Low-Level Light Therapy.

\section{Resumen}

La sífilis congénita sigue siendo una realidad en los países del tercer mundo, lo que genera complicaciones locales Y sistémicas que comprometen la calidad de vida del paciente. Así, el objetivo de este artículo es presentar el caso clínico de una paciente lactante de 34 días con sífilis congénita. En el examen clínico, el paciente presentaba numerosas ulceraciones Y lesiones tisulares en boca, cara Yextremidades. La terapia adyuvante, los enfoques estándar se han asociado con la aplicación de láser de baja potencia. Luego de 8 sesiones de terapia fotodinámica, hubo remisión completa del cuadro clínico presentado, devolviendo la calidad a la tierna vida del paciente. Con el caso presentado, las manifestaciones Y complicaciones de la sífilis siguen siendo un desafío. Es fundamental que el equipo multidisciplinario pueda diagnosticar Y tratar la sífilis congénita lo antes posible. El odontólogo debe conocer las diversas manifestaciones clínicas del mismo para ayudar en el correcto diagnóstico Y tratamiento antes de la aparición de los síntomas. La terapia de fotobiomodulación con láser de baja potencia pareció acelerar satisfactoriamente el proceso de reparación de tejidos. La respuesta del paciente al tratamiento instituido fue gratificante.

Palabras clave: Sífilis; Sífilis congénita; Terapéutica; Terapia por Luz de Baja Intensidad.

\section{Introdução}

A sífilis é uma infecção sistêmica, causada pela bactéria Treponema pallidum, que pode ser transmitida de forma transversal, da gestante ao bebê, causando a sífilis congênita (SC). O controle da SC é importante pois continua sendo uma doença impactante na saúde pública, causadora de mortes fetais e neonatais, prematuridade, baixo peso ao nascer e manifestações graves com sequelas irreversíveis (Domingues \& Leal, 2016; Gomez et al, 2013; Rocha, 2021).

Dados do SINAN, Sistema de Informações de Agravos de Notificação, entre 1998 e junho de 2020, apresentam 236.355 casos de sífilis congênita em menores de um ano de idade. Os dados mais recentes do Brasil referentes a sífilis congênita mostram que a taxa de incidência é de 8,2/1.000 nascidos vivos e a taxa de mortalidade é 5,9/100.000 nascidos vivos, ambos no ano de 2019. As regiões Norte e Centro-Oeste tiveram discreto aumento no número de casos notificados: em 2019, ambas apresentaram aumento de 0,3\% em relação a 2018. No ano de 2013 o Mato Grosso do Sul, estado da região Centro-Oeste, apresentou a maior taxa entre todas as Unidades da Federação: 16,7 casos/1000 nascidos vivos. Entretanto, em 2019 o estado apresentou uma média de 7 casos/1000 nascidos vivos e não é mais o estado com a maior taxa (Brasil, 2013; Brasil, 2020).

As manifestações da SC até os 2 anos de idade são consideradas precoces ou após essa idade como SC tardia. Geralmente os sintomas estão relacionados com alterações dermatológicas, oftalmológicas, ósseas, auditivas, odontológicas e neurológicas. A divulgação das complicações e manifestações clínicas que a sífilis congênita provoca é muito relevante diante do cenário atual da infecção no mundo, assim como a identificação dos aspectos relacionados a prevenção e quanto ao tratamento da SC (Rocha, 2021; WHO, 2016).

Posto isto, o tratamento padrão mesmo que depende do estágio da doença, tem a penicilina como protocolo preconizado, desde que nenhuma alergia seja associada. Quando da presença de manifestações orais ulcerosas, o uso do laser de baixa potência pode ser considerado como recurso terapêutico adjuvante, uma vez que é não invasivo e de baixo custo (Hertel et al, 2014; Giovani et al, 2012).

O uso da fotobiomodulação com o laser de baixa potência (LBP) pode atuar como terapia adjuvante a aceleração da cicatrização e melhora do controle álgico, culminando no aumento da qualidade vida, diminuição do tempo de internação hospitalar e riscos de infecções secundárias, uma vez que as úlceras serão mais rapidamente cicatrizadas (Oliveira et al, 2020).

Logo, o objetivo deste trabalho é através da descrição de um caso clínico de paciente diagnosticado com sífilis congênita, cujo uso adjuvante da fotobiomodulação com o laser de baixa potência nas lesões ulcerosas obteve sucesso, com rápida cicatrização, contribuindo de forma efetiva para o acervo bibliográfico científico do tema, em específico quanto as suas 
manifestações odontológicas e o tratamento utilizado.

\section{Metodologia e Relato de Caso}

O presente caso trata-se de um relato com fins descritivos, exploratórios e com abordagem qualitativa, a fim de mostrar sua relevância clínica e facilitar pesquisas e novos relatos com a mesma temática, sempre baseada em evidência, usando como base referencial Pereira (2018) e Souza et al. (2021). O trabalho recebeu anuência dos genitores do paciente após assinatura do Termo de Consentimento Livre e Esclarecido para participação. Foram respeitados todos os princípios éticos na condução do tratamento do paciente. Assim, este trabalho faz menção a um paciente do sexo masculino, com 34 dias de vida, lactante, internado no Hospital Universitário Maria Aparecida Pedrossian/HUMAP-UFMS da cidade de Campo Grande (Mato Grosso do Sul). A mãe relata que com cerca de 11 dias após o nascimento, o recém-nascido apresentou perda ponderal e pouca aceitação da dieta, quando aquela buscou atendimento médico.

$\mathrm{Na}$ consulta médica foi prescrito uma fórmula nutricional de $30 \mathrm{~mL}$ ofertada a cada 3 horas, mas, com moderada aceitação pelo lactante. Após iniciar o uso, evoluiu para um quadro de diarreia (fezes aquosas, sem muco, sem sangue, mais de 5 vezes ao dia) sendo necessário a substituição da fórmula, mas sem sucesso, ainda sendo constante o quadro de evacuação.

Após 1 dia, evoluiu com febre não aferida, lesões em pele e sonolência buscando novamente o atendimento médico que encaminhou o paciente para à atendimento hospitalar sendo realizados exames de sangue, do líquido cefalorraquidiano e radiografia de tórax. Desde já, começou-se o uso de Ampicilina 66,5mg/kg/dose, gentamicina 4mg/kg/dose, Penicilina Procaína e transfusão de $40 \mathrm{ml}$ de concentrados de hemácia.

Neste momento, o paciente foi avaliado pela Odontologia Hospitalar do HUMAP/UFMS, pois, ele apresentava lesões em caráter sanguinolento e crosta na região de lábios superior e inferior, além de lesões em mucosa interna, mãos e pés, ambas se apresentavam sanguinolentas e em aspecto de crosta. Em conjunto multiprofissional foram elencados diagnóstico e plano de tratamento ao paciente. Primeiramente, evidenciou tratar-se de um caso de sífilis congênita, sendo assim, os pais foram tratados devidamente, e começou a ser administrado penicilina cristalina $200.000 \mathrm{UI} / \mathrm{kg} / \mathrm{dia}$ e dado continuidade ao tratamento de suporte ao paciente associado a tratamento das lesões intra e peribucais (Figura 1). 
Figura 1: Lesões ulceradas e crostosas em região peribucal e membros, superiores e inferiores.

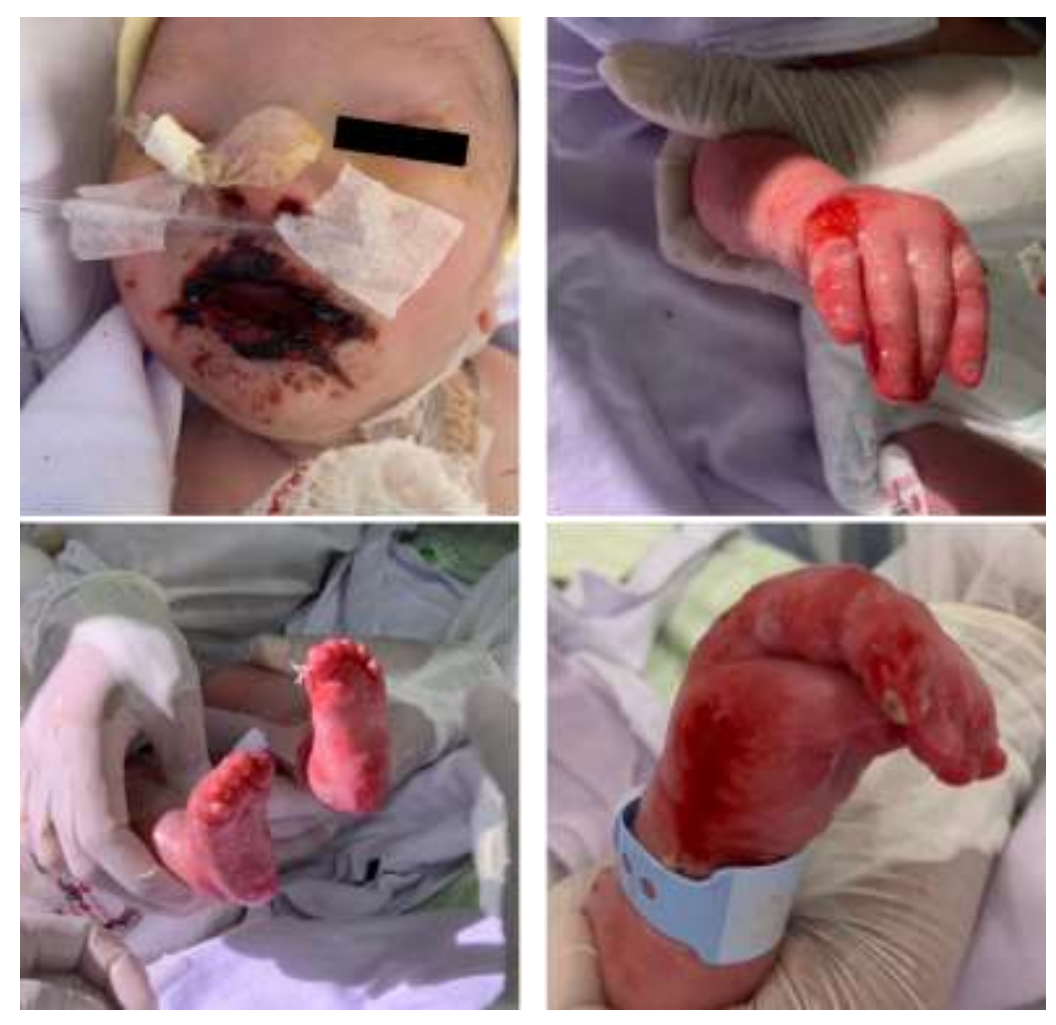

Fonte: Autores.

Para tanto, foi iniciada a terapia de fotobiomodulação (PBMT), visando biomodulação tecidual por segunda intenção. Durante a primeira e segunda sessões foi utilizado o laser de baixa potência em contato com os tecidos. Cada área de $1 \mathrm{~cm} 2$ foi irradiada por 10 segundos, com $100 \mathrm{~mW}$ de potência e $6 \mathrm{~J}$ de energia a fim de diminuir edema em lábios e língua para que fosse conseguido o acesso a cavidade bucal. Para aPDT (terapia fotodinâmica antimicrobiana) foi utilizado 0,01\% de azul de metileno aplicado nas lesões e permanecendo por 5 minutos (tempo pré-irradiação) e então foram utilizados os mesmos parâmetros para o laser, porém, com o aumento para 90 segundos, ou seja, 9J por lesão. No primeiro dia de condução da proposta terapêutica, o aPDT foi realizado concomitantemente com a PBMT, sendo que apenas a PBMT repetida a cada 24 horas, por 8 dias, com a diminuição da energia de irradiação que foi ajustada para 3J por ponto de lesão.

Agentes tópicos adjuvantes às sessões de laserterapia foram precedidas de higienização com soro fisiológico e uso de óleo hidratante (AGE - agentes graxos essenciais) com intuito de remover a porção mais externa das crostas corporais, peribucais, e realizar a penetração mais intensa do laser.

O aspecto apresentava-se melhor após a 4a sessão, sendo assim, optou-se em diminuir para o intervalo de 24 horas, com a mesma frequência. Após a 6 sessão, conseguimos observar um padrão de reparação em cerca de 80 \% das lesões, chegando ao aspecto rosado e natural da pele, ausência de sangramento e com fator de cicatrização otimizado, seguimos até a 8 sessão com sucesso do tratamento. Em todas as sessões foram feitas a limpeza do local, e a aplicação do laser de forma apresentada acima, mudando apenas o intervalo entre as sessões (Figura 2). 
Figura 2: Melhora do aspecto das lesões cutâneas e peribucais até completa remissão das mesmas ao final da terapia fotodinâmica. Quatro e oito sessões de aplicação do laser de baixa potência, respectivamente.
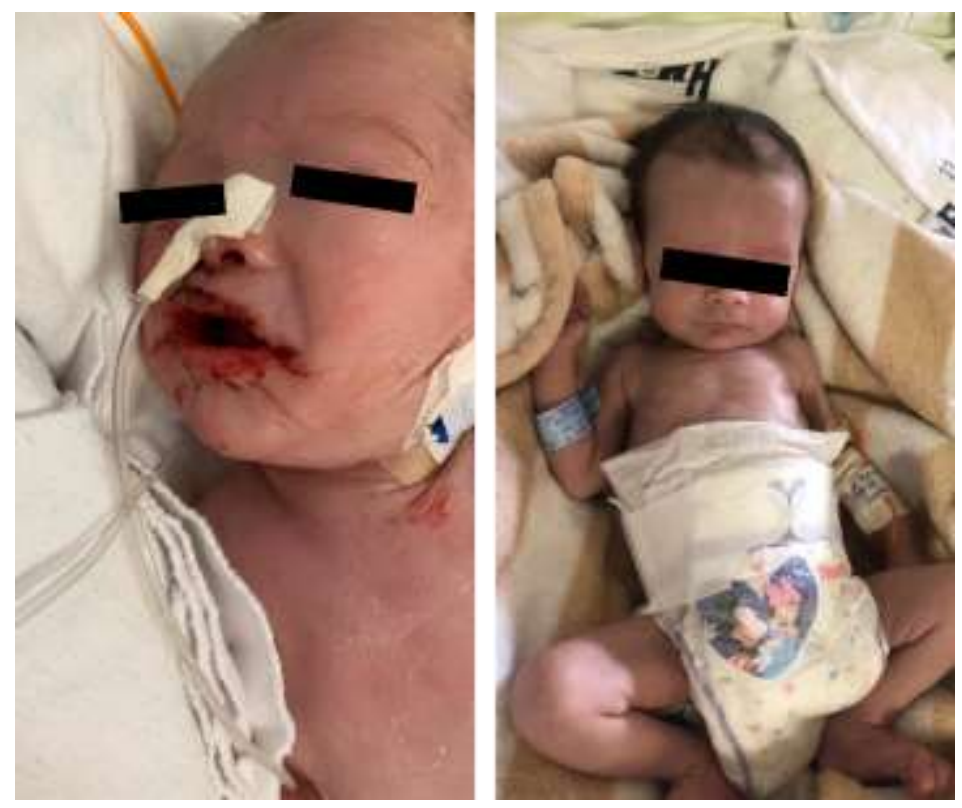

Fonte: Autores.

\section{Discussão}

A sífilis ainda é uma realidade em nosso país e em muitos países socio e economicamente comprometidos. Vem ganhando altas proporções na última década, talvez relacionada a ausência de ações preventivas ou baixa adesão às ações existentes, por parte da população (Ribeiro et al, 2020; Brasil, 2019; Brasil, 2020).

Neste sentido, a busca pelo diagnóstico precoce quando da manifestação da doença é essencial para o melhor plano de tratamento. As lesões bucais da sífilis facilitam o diagnóstico da doença fato evidenciado pelo caso clínico acima. As inúmeras lesões da sífilis congênita recente, descritas no caso, mostram como é mandatória a avaliação clínica do cirurgião-dentista no atendimento integrado em ambiente hospitalar, já que o paciente apresentou inúmeras soluções de continuidade que se coalesceram resultando em grande perda tecidual em boa parte do corpo, região de mucosa oral e perilabiais, sangrantes ao toque e que podem gerar uma sintomatologia dolorosa intensa.

Os tratamentos empregados às lesões descamativas e ulceradas visam a melhora da qualidade de vida do paciente, acima de tudo, com manutenção ou restabelecimento de uma saúde bucal e, por que não, sistêmica, com alívio da sintomatologia dolorosa e retorno à função normal. Isso se torna mais evidente quando o indivíduo portador das manifestações orais e peribucais é um recém-nascido, que com dificuldade de externar seus incômodos e anseios tornam o tratamento ainda mais desafiador. Assim, a busca de meios alternativos ou complementares como o laser de baixa potência se torna crucial para otimizar os resultados.

As terapêuticas tópicas preconizadas em lesões ulceradas compreendem o uso do Sulfato de Neomicina com Tartarato de Bismuto (Barros et al, 2021), Acetonida de Triancinolona (Barros et al, 2021), Sulfadiazina de Prata, Ácidos Graxos Essenciais, hidrocoloides e óleos minerais (Brasil, 2002). Atualmente, vem ganhando grande aceitação, com resultados muito positivos, o uso do laser de baixa potência. No caso apresentado, além da limpeza cuidadosa das regiões acometidas com soro fisiológico $0,9 \%$ para remoção cuidadosa, lenta e graduação das crostas, o laser foi fundamental na reparação tecidual. A potencialização do processo de reparo com o laser se mostrou evidente tanto no controle do processo inflamatório, neoformação vascular e proliferação tecidual como bem demonstrado por Dias de Andrade et al. (2014) além de inibir o 
crescimento microbiano (Lima et al., 2020).

A utilização do laser na diminuição da sintomatologia dolorosa relacionada a lesões de perda de continuidade tecidual também é demonstrada pela literatura, seja associado ou não a outras terapêuticas (Macedo et al., 2021; Raffaele et al, 2020; Raffaele et al, 2021). Com o protocolo da laserterapia empregado após a quarta sessão houve melhora significativa sobretudo das lesões bucais e peribucais devolvendo ao paciente aspecto tecidual normal e qualidade de vida.

\section{Considerações Finais}

A sífilis congênita apresenta uma grande quantidade de manifestações clínicas, mas na maioria dos casos pode ser sutil e inespecífica. Os sinais e sintomas podem surgir precocemente (em até dois anos após o nascimento) ou tardiamente (após o período de 2 anos). O caso de um recém-nascido que apresentou descamação cutânea acral e fissuras periorais (rágades), em cavidade oral, extremidades como mãos/pés o que evidenciou fortemente as características da sífilis congênita precoce.

Portanto, é importante o cirurgião-dentista ter o conhecimento das diversas manifestações clínicas da sífilis congênita precoce para ajudar no diagnóstico correto e tratamento antes do começo de sinais e sintomas. Além disso, usar medidas terapêuticas alternativas úteis como a terapia de fotobiomodulação com laser de baixa potência para acelerar o processo de cicatrização das feridas causadas pela sífilis e, assim, colaborar para a boa evolução do quadro clínico e a diminuição do tempo de internação hospitalar.

Este artigo guarda as limitações inerentes a um relato de caso. Assim sendo, novos trabalhos devem ser realizados a fim de aumentar a confiabilidade estatística dos resultados alcançados e facilitar o atendimento do paciente nas mesmas condições aqui descritas.

\section{Agradecimentos}

O presente trabalho foi realizado com apoio da Fundação Universidade Federal de Mato Grosso do Sul UFMS/MEC- Brasil.

\section{Referências}

Barros, B. F. M., Costa, C. M., Mattos, G. M. L. et al. (2021). Uso do laser de baixa potência no tratamento de lesões bucais em pacientes com doenças neurológicas: Relato de caso. Research, Society and Development. 10(6), e47110616083.

Brasil. (2019). Ministério da Saúde. Secretaria de Vigilância em Saúde. Departamento de Doenças de Condições Crônicas e Infecções Sexualmente Transmissíveis. Protocolo Clínico e Diretrizes Terapêuticas para Prevenção da Transmissão Vertical do HIV, Sífilis e Hepatites Virais. Brasília: Ministério da Saúde.

Brasil. (2020). Ministério da Saúde. Secretaria de Vigilância em Saúde. Boletim Epidemiológico Especial. Súfilis.

Brasil. (2002). Ministério da Saúde. Secretaria de Políticas de Saúde. Departamento de Atenção Básica. Manual de condutas para úlceras neurotróficas e traumáticas / Ministério da Saúde, Secretaria de Políticas de Saúde, Departamento de Atenção Básica. - Brasília: Ministério da Saúde.

Dias de Andrade, F. S. S., Clark, R. M. O. \& Ferreira, M. L. (2014). Efeitos da laserterapia de baixa potência na cicatrização de feridas cutâneas. Revista do Colégio Brasileiro de Cirurgĩ̃es. 41(2): 129-133.

Domingues, R. M. S. M. \& Leal, M. C. (2016). Incidencia de sifilis congenita e fatores associados a transmissao vertical da sifilis: dados do estudo Nascer no Brasil. Caderno de Saúde Pública. 32(6):e00082415.

Giovani, E. M., De Paula Neto, E. R., Vieira, B. C., \& De Andrade, D. M. (2012). Conventional systemic treatments associated with therapeutic sites of local lesions of secondary syphilis in the oral cavity in patients with AIDS. Indian journal of dental research: official publication of Indian Society for Dental Research. 23(5), 670-673.

Gomez, G. B., Kamb, M. L., Newman, L. M., Mark, J., Broutet, N., \& Hawkes S. J. Untreated maternal syphilis and adverse outcomes of pregnancy: asystematic review and meta-analysis. Bulletin of the World Health Organization. 2013; 91:217-26.

Hertel, M., Matter, D., Schmidt-Westhausen, A. M. \& Bornstein, M. M. (2014). Oral syphilis: a series of 5 cases. Journal of oral and maxillofacial surgery: official journal of the American Association of Oral and Maxillofacial Surgeons. 72(2), 338-345. 
Lima, A. M. C. T., Sergio, L. P. S., Trajano, L. A. S. N. et al. (2020). Photobiomodulation by dual-wavelength low-power laser effects on infected pressure ulcers. Lasers in Medical Science. 35:651-660.

Macedo, S. P. R., De Almeida Mota, M. S., Fagundes, C. F., De Souza, M. R., \& Navarro, R. S. (2021). Efeitos da fotobiomodulação no tratamento de úlceras por pressão: Revisão integrativa. Research, Society and Development. 10(2), e32810212597.

Ministério da Saúde (BR). (2015). Secretaria de Vigilância em Saúde. Departamento de DST, Aids e Hepatites Virais. Sífilis. Boletim Epidemiológico.

Ministério da Saúde (BR). (2020). Secretaria de Vigilância em Saúde. Departamento de DST, Aids e Hepatites Virais. Sífilis. Boletim Epidemiológico.

Oliveira, C. G., Freitas, M. F., De Sousa, M., Giorgi, R., \& Chacur, M. (2020). Photobiomodulation reduces nociception and edema in a CFA-induced muscle pain model: effects of LLLT and LEDT. Photochemical \& photobiological sciences : Official journal of the European Photochemistry Association and the European Society for Photobiology. 19(10), 1392-1401.

Raffaele, R. M., Baldo, M. E., Queiroz, P. F. S., Tateno, R. Y., Palma L. F., \& Campos, L. Phototherapies in the management of an ulcerative lip lesion in a patient taking multiple medications for rheumatoid arthritis. Research, Society and Development. 10(8), e50210817538.

Raffaele, R. M., De Oliveira, R. L., Baldo, M. E., Santana, G. U., \& Pacheco, J. A. (2020). Treatment of Low Level Laser Therapy in the Oral Manifestations of Steven Johnson Syndrome. International Journal of Emergency Medicine. 1(3):1-8.

Ribeiro, R. S., Segura, G. S., Ferreira, A. C. M. et al. (2020). Epidemiologia da sífilis gestacional e congênita: revisão integrativa de literatura. Research, Society and Development. 9(4), e178942470.

Rocha, A. F. B., Araujo, M. A. L., Barros, V. L., Americo, C. F., \& Silva Jr, G. B. (2021). Complications, clinical manifestations of congenital syphilis, and aspects related to its prevention: an integrative review. Revista Brasileira de Enfermagem. 74(4):e20190318.

Souza, Y. D., Seco, A. J. L. G., Silva, J. C. L. Da., Pereira, K. F. S., \& Jardim, E. C. G. (2021). Kaposi’s Sarcoma in an HIV and Covid-19 positive patient. Research, Society and Development. 10(11), e19101119149.

World Health Organization (WHO). (2016). Guidelines for the treatment of Treponema pallidum (syphilis). WHO. 